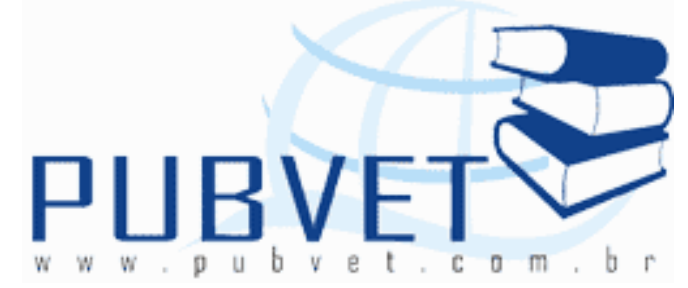

PUBVET, Publicações em Medicina Veterinária e Zootecnia.

\title{
Estudo dos elementos vasculares, arteriais e venosos do hilo renal em javali Sus scrofa scrofa ( Linnaeus, 1758)
}

André Luiz Quagliatto Santos, Lucélia Gonçalves Vieira, Líria Queiroz Luz Hirano, Árthur Paulino Sanzo Kaminishi, Juliana dos Santos Mendonça, Thaís Carneiro Santos Rodrigues, Sthéfani Evangelista Siqueira

Laboratório de Ensino e Pesquisa em Animais Silvestres (LAPAS), Universidade Federal de Uberlândia (UFU). Avenida Amazonas 2245, Jardim Umuarama, 38.405-302, Uberlândia-MG.

E-mail: quagliatto@famev.ufu.br

\section{Resumo}

Informações científicas encontradas na literatura sobre javalis se referem mais especificamente a aspectos comportamentais, zoológicos e reprodutivos sendo escasso estudos envolvendo sua morfologia. Com o intuito de contribuir com o manejo e a conservação desses animais, foi estudado análises de variações anatômicas no comportamento dos vasos do hilo renal na espécie Sus scrofa scrofa. Foram utilizados assim 13 pares de rins de javalis, provenientes de um criatório comercial no município de Romaria - MG. Estes foram fixados em formol a $10 \%$ e posteriormente foram removidos em conjunto com os correspondentes segmentos da aorta, veia cava caudal e trato inicial do ureter. Foi realizada a dissecação do material e análise com o auxilio de uma lupa tipo Wild $10 \mathrm{X}$. De acordo com os resultados encontrados, observou-se que $\mathrm{o}$ número de ramos da artéria renal esquerda varia de 3 a 5 , sendo a maior 
frequência de 3 ramos com $38,46 \%$ e que na artéria renal direita variou entre 2 e 6 , sendo 4 ramos e a maior frequência com 30,77\%. O número de raízes venosas esquerdas variou entre 2 e 4 , sendo 2 a maior frequência com $84,60 \%$ e nas direitas variou entre 2 e 3 sendo a maior frequência de 2 raízes com 76,92\%. A maior frequência de ramos arteriais direitos e esquerdos e raízes venosas direitas e esquerdas foram encontradas no quadrante II.

Palavras-chave: anatomia, hilo renal, javalis, rim.

\title{
Study of vascular elements, arterial and venous hilum in wild boar Sus scrofa scrofa (Linnaeus, 1758)
}

\begin{abstract}
Information found in the scientific literature on boars refer specifically to behavioral, reproductive and zoologically aspects, being scarce studies involving morphology. In order to contribute to the management and conservation of these animals, we analysed anatomical variations in the behavior of vessels in the Sus scrofa scrofa hilus. We used 13 pairs of kidneys from wild boars from a commercial breeding facility in the Pilgrimage - MG city. These were fixed in formalin $10 \%$ and subsequently were removed together with the corresponding segments of the aorta, cava caudal vena and initial ureter tract. We performed the analysis and dissection of the material with the aid of a magnifying glass 10 Wild type X. According to the results, it was observed that the number of branches of the left renal artery ranges from three to five, with the highest frequency of three branches with $38.46 \%$ and that in right renal artery ranged between two and six, and four branches and more frequently with $30.77 \%$. The number of the left venous roots ranged between two and four, with two roots the higher frequency $(84.60 \%)$. In the right side, the values raged between two and three, whit two roots the higher frequency $(76.92 \%)$. The higher frequency of arterial the right and left branches and right and left venous roots were found in quadrant II.
\end{abstract}

Keywords: anatomy, renal hilum, boars, kidney. 


\section{INTRODUÇÃO}

O javali é um suídeo silvestre nativo do continente africano (ANTUNES, 2001). São considerados os mais autênticos ancestrais dos nossos porcos domésticos (CANEJO; CORREIA FILHO, 2002). Eles foram trazidos para a Argentina com fins de caça esportiva e acabaram se espalhando pelo sul do Brasil. Em função de sua alta adaptabilidade, é criado com êxito, em todos os continentes, visando a atividadede caça esportiva e, principalmente, produção de carne, muito apreciada por ser saudável, exótica e natural (PRADA, 2004; CARVALHO et al., 2006). A sua carne é apreciada pelo homem, graças à sua qualidade, apresentando baixos teores de gordura, colesterol e poucas calorias, tendo alto teor protéico quando comparada com a de bovino, despertando o interesse dos nutricionistas e consumidores (ANTUNES, 2001). No Brasil, existem criatórios comerciais de javalis e é uma atividade em expansão (CANEJO; CORREIA FILHO, 2002).

Os rins são orgãos de consistência firme, e em suínos são mais achatados do que em bovinos, sendo raramente simétricos, e apresentam cor castanho-avermelhada (DYCE et al., 1997). É um dos órgãos responsáveis pela homeostasia, filtrando o plasma. O estudo da morfologia dos rins constitui-se em um assunto que se torna cada vez mais de grande relevância pela sua importância funcional e pelas variações relativas à sua organização estrutural nas diferentes espécies animais. A segmentação anatômica pauta-se no interesse da cirurgia experimental, particularmente nas ressecções parciais do órgão (DIDIO, 1985).

Em pesquisas sobre artérias renais, sobretudo em roedores, observou-se que a maioria dos autores não identifica os segmentos anátomo-cirúrgicos renais nos animais (SALVINI et al., 1981, CHAVEZ et al., 1982, VILARTA et al., 1982, NEVES et al., 1993, BARONE et I., 1997). Já em suiformes como a queixada (Tayassi pecari), por exemplo, os setores vasculares renais foram 
SANTOS, A.L.Q. et al. Estudo dos elementos vasculares, arteriais e venosos do hilo renal em javali Sus scrofa scrofa ( Linnaeus, 1758). PUBVET, Londrina, V. 7, N. 10, Ed. 233, Art. 1541, Maio, 2013.

definidos e denominados de acordo com sua disposição topográfica (ROMAGNOLLI et al., 2003).

As informações científicas encontradas sobre javali se referem mais especificamente a aspectos comportamentais, zoológicos e reprodutivos Porém, a literatura apresenta-se bastante escassa em termos morfológicos, sendo encontrados trabalhos com outros animais da superfamília suidoidae, como suíno (FAGUNDES, 1984; SOUZA et al., 1984; COSTA et al., 1991; EVAN et al., 1996; SAMPAIO et al., 1996; SOUZA, 1997) e cateto (MACHADO et al., 2000), referindo-se a distribuição ou segmentação arterial no rim destes espécimes. Assim, visando o manejo e a conservação desses animais, propusemo-nos a buscar variações anatômicas no comportamento dos vasos do hilo renal, fornecer subsídios para trabalhos de natureza cirúrgicoexperimental e ainda contribuir para o desenvolvimento da anatomia comparada.

\section{MATERIAL E MÉTODOS}

Foram utilizados, nesta pesquisa, 13 pares de rins de javalis, sendo 05 fêmeas e 08 machos provenientes de um criatório comercial no município de Romaria - MG, fixados em formol a $10 \%$ por período superior a 7 dias. Posteriormente foram removidos os rins em conjunto, com os correspondentes segmentos da aorta, veia cava caudal e trato inicial do ureter, dissecaram-se as artérias e veias do pedículo renal retirando-se o tecido adiposo que envolve estes vasos. Os rins foram analisados com o auxilio de uma lupa tipo Wild 10 $X$. Para análise dos resultados, foram construídos desenhos esquemáticos, como os usados em suínos (FERREIRA, 1975), dividindo o hilo renal em quadrantes, mediante duas linhas imaginárias perpendiculares: a primeira no sentido craniocaudal, traçada de pólo a pólo, passando pelo centro do ureter e a segunda, no sentido ventrodorsal, interseccionando a primeira, de modo a dividir o segmento hilar em duas metades, delimitando os quadrantes I, II, III 
e IV, que por sua posição, denominou-se de craniodorsal (CrD), crânioventral $(\mathrm{Crv})$, caudodorsal $(\mathrm{CaD})$ e caudoventral $(\mathrm{CaV})$, respectivamente.

\section{RESULTADOS E DISCUSSÃO}

Observou-se que o número de ramos da artéria renal esquerda varia de 3 a 5, sendo a maior frequência de 3 ramos com 38,46\%. Na artéria renal direita o número de ramos variou entre 2 e 6 , sendo 4 ramos e a maior frequência com $30,77 \%$. O número de raízes venosas esquerdas variou entre 2 e 4, sendo 2 a maior frequência com $84,60 \%$. O número de raízes venosas direitas variou entre 2 e 3 sendo a maior frequência de 2 raízes com 76,92\%. A maior frequência de ramos arteriais direitos e esquerdos e raízes venosas direitas e esquerdas foram encontradas no quadrante II, com $43,48 \%$ e $33,33 \%, 53,60 \%$ e $42,86 \%$ respectivamente. Os ramos da artéria renal esquerda se dirigem na sua maior parte para o quadrante IV frequência de $40 \%$. O quadrante II é o mais povoado pelos ramos da artéria renal direita, também com frequência de $40 \%$. As raízes da veia renal esquerda e direita emergem em sua maioria do quadrante II de seus respectivos rins na frequência de 53,84\%. Foram encontradas na intersecção dos quadrantes I e II, III e IV, uma e duas raízes venosas esquerdas respectivamente e uma na intersecção dos quatro quadrantes.

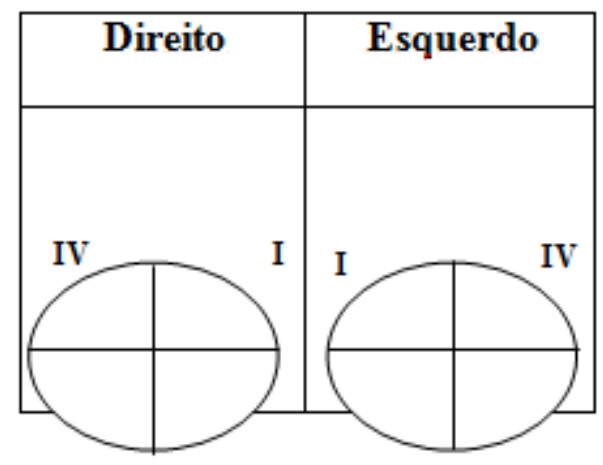

Figura 1: Disposição dos quadrantes craniodorsal, craniventral, caudodorsal e caudoventral (I, II, III e IV), respectivamente. 
Diferentemente do encontrado no presente trabalho, em suínos da raça Large White e Landrace (COSTA et al., 1991; SOUZA et al., 1984), a artéria renal esquerda varia de 4 a 18 ramos e 5 a 13 ramos, apresentando maior freqüência dos ramos 10 e 8, emitindo o maior numero de ramos no quadrante I e II, respectivamente. Na artéria renal direita apresenta variação de 6 a 20 e 4 a 16 e freqüências de 10 e 6,7,8 respectivamente, emitindo maior numero de ramos no quadrante I. A veia renal direita sofre variação de 1 a 5 e 2 e 4, com maior freqüência de 5 raízes e 2 respectivamente. A veia renal esquerda apresenta variação entre 2 e 7 raízes e 1 e 5 com maior freqüência o números de 3,4 e 2 respectivamente. Apresentando maior numero de ramos quadrante II.

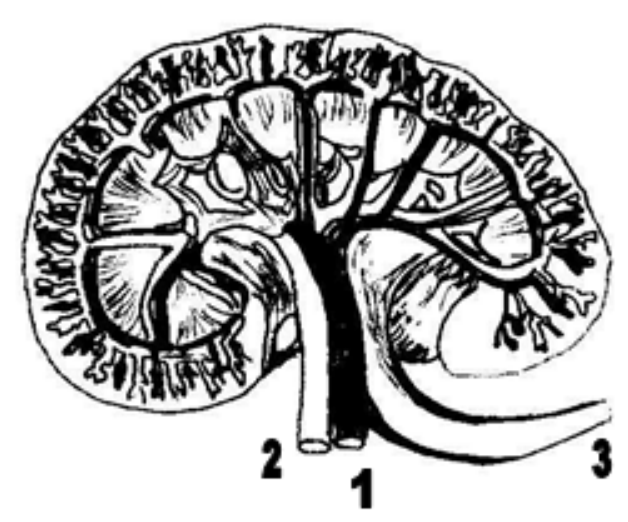

Figura 2: Desenho de rim de Sus scrofa scrofa, mostrando artéria renal e seus ramos (1), veia renal e seus ramos (2) e ureter (3).

Em estudos de Romagnolli et al., (2003) com queixadas, verificaram que a artéria renal esquerda emitiu três ramos setoriais (88,88\%): cranial, dorsal e ventral $(66,66 \%)$ e dorsal, ventrocranial e ventrocaudal $(11,11 \%)$; quatro ramos setoriais: dorsocranial, dorsocaudal, ventrocranial e ventrocaudal $(11,11 \%)$. A artéria renal direita bifurcou-se em ramos setoriais renais cranial e caudal (5,55\%); trifurcou-se em ramos cranial, dorsal e ventral $(33,33 \%)$, ramos ventral, dorsocranial e dorsocaudal (16,66\%), ramos.

Quanto ao comportamento da artéria renal, sempre única e ramificada, os resultados desta pesquisa coincidem com aqueles expostos em catetos 
SANTOS, A.L.Q. et al. Estudo dos elementos vasculares, arteriais e venosos do hilo renal em javali Sus scrofa scrofa ( Linnaeus, 1758). PUBVET, Londrina, V. 7, N. 10, Ed. 233, Art. 1541, Maio, 2013.

(MACHADO, G. V. et al. 2000b), em queixadas ( MACHADO, G. V. et al., 2000a; ROMAGNOLLI, 2003), em suínos (SOUZA, 1997; EVAN, 1996, SAMPAIO et al., 1996; FAGUNDES, 1984, FERREIRA, 1975); em caprinos mestiços (CARVALHO, 1995); em pequenos ruminantes(CARVALHO; PEREIRA, 1987; EL-KHALIGI, et al., 1987; MIGLINO, et al. 1985; PEDUTI-NETO, 1976; ALBUQUERQUE, 1979); em cães (CORREIRA; ESPERANÇA-PINA， 1982; ARNAUTOVIC, 1959).

Para rins de catetos (Tayassu tajacu), Machado et al., (2000b) descrevem a ocorrência da artéria setorial dorsal, dosrocranial, dorsocaudal, vetral, ventrocranial, ventrocaudal e caudal para o rim esquerdo. Sendo que o rim direito apresentou, como ramos primários da artérias renal, as artérias setoriais cranial, dorsal, dorsocaudal, dorsocranial, ventral, ventrocranial, ventrocaudal e caudal. Resultados semelhantes foram encontrados por Romagnolli, et al., (2003). No entanto, não foi o objetivo desse trabalho descrever os mesmos ramos.

Os resultados em suínos demonstraram divisão das artérias renais, direita e esquerda, somente em ramos setoriais craniais e caudais, sendo que em todos eles, estão ausentes os ramos setoriais dorsal, dorsocranial, dorsocaudal, ventral, ventrocranial e ventrocaudal (SOUZA, 1997; EVAN et al., 1996; SAMPAIO et al., 1996; FAGUNDES, 1984; FERREIRA, 1975). Resultados semelhantes foram encontrados em queixadas, de acordo com ROMAGNOLLI et al., (2003).

Em caprinos mestiços, Carvalho(1995) descreveu a divisão da artéria renal tanto esquerda quanto direira, em ramos setoriais, ventral e dorsal. Em cães, Correia e Esperança-Pina (1982) citaram a existência de ramos setoriais, dorsal e ventral, semelhante ao que foi citado para pequenos ruminantes.

\section{CONCLUSÕES}

Observou-se que o número de ramos da artéria renal esquerda varia de 3 a 5, sendo a maior freqüência de 3 ramos com 38,46\% e que na artéria 
SANTOS, A.L.Q. et al. Estudo dos elementos vasculares, arteriais e venosos do hilo renal em javali Sus scrofa scrofa ( Linnaeus, 1758). PUBVET, Londrina, V. 7, N. 10, Ed. 233, Art. 1541, Maio, 2013.

renal direita variou entre 2 e 6 , sendo 4 ramos e a maior freqüência com $30,77 \%$. O número de raízes venosas esquerdas variou entre 2 e 4 , sendo 2 a maior freqüência com $84,60 \%$ e nas direitas variou entre 2 e 3 sendo a maior freqüência de 2 raízes com 76,92\%. A maior freqüência de ramos arteriais direitos e esquerdos e raízes venosas direitas e esquerdas foram encontradas no quadrante II.

\section{REFERÊNCIAS BIBLIOGRÁFICAS}

ALBUQUERQUE, J. F. C. Contribuição ao estudo da vascularização arterial do rim em caprinos (Capra hircus Linnaeus, 1758). 1979. 93 f. Tese (Doutorado em Anatomia dos Animais Domésticos). Universidade de São Paulo, Faculdade de Medicina Veterinária e Zootecnia, São Paulo, 1979.

ANTUNES, R. Comida de gaulês? Suin. Industr., v.151, p. 24-27, 2001.

ARNAUTOVIC, I. The distribution of the renal artery in the kidney of the dog. British Veterinary Journal, London, v. 115, n. 13, p. 446-448, 1959.

BARONE R. 1997. Anatomie comparée dés mammiféres domestiques. Vol.3. $2^{\mathrm{a}}$ ed. Vigot, Paris, p.130.

CANejO, M.; CORReia filho, J. A nobreza do Rústico. Panorama Rural, São Paulo, no 36, p. 40-45, 2002.

CARVALHO, M. A. M. et al., Segmentação anatomo-cirúrgica arterial do rim de javali (Sus scrofa) adulto. Biotemas, v. 4, p. 85-90, 2006.

CARVALHO, M. A. M. Segmentos anatomocirúrgicos arteriais do rim de caprinos mestiços. 1995. 95 f. Tese (Doutorado) - Universidade de São Paulo, Faculdade de Medicina Veterinária e Zootecnia, São Paulo, 1995.

CARVALHO, M. A. M.; PEREIRA, J. G. L. Contribuição ao estudo dos elementos vasculares, arteriais e venosos, hilares e extra-hilares, em rins de caprinos da raça Moxotó. Rev. Fac. Med. Vet. Zoot. Univ. São Paulo, v. 24, n. 2, p. 119-126, 1987.

CHAVEZ W. R. L. et al., 1982. Contribuição ao estudo dos elementos vasculares, arteriais e venosos, hilares e extrahilares, do hilo renal no hamster dourado (Mesocricetus auratus). Anais $1^{\text {a }}$ Sem. Vet. Fac. Med. Vet. Zootec. USP, São Paulo, p.24.

CORREIA, M.; ESPERANÇA-PINA, J. A. Territórios arteriais no rim do cão. Arquivos de Anatomia e Antropologia, v. 6/7, p. 113-127, 1982.

COSTA, O. M. et al., Contribuição ao estudo dos elementos vasculares, arteriais e venosos, do hilo renal de suínos (Sus scrofa domestica - Linnaeus - 1758) da raça Large- While. Brazilian Journal of Veterinary Research and Animal Science, v. 28, p. 55-69, 1991. 
COSTA, O.M.; PEREIRA, J.G.L.; RODRIGUES, C.A. Contribuição ao estudo dos elementos vasculares, arteriais e venosos do hilo renal em suínos (Sus scrofa domestica - Linnaeus 1758) da raça Large White. Braz. J. vet. Res. Anim. Sci., São Paulo, v. 28, no 2, p. 155-69, 1991.

DIDIO, L.J.A. 1985. Segments of the kidney: the anathomical basis for nephrosegmentectomy, p.1-2. In: DiDio L.J.A. \& Motta P. (ed.), Basis of Clinical, and Surgical Nephrology. Martins Nijhoff, Boston.

EL-KHALIGI, G. M. et al. Gross anatomical studies on the renal arteries of same domestic animals. Part II: sheep. Vet. Med. Jour. v. 35, n. 1, p. 7-15, 1987.

EVAN, A. P. et al., Branchin patterns of the renal artery of the pig. The Anatomical Record, v. 246, p. 217-223, 1996.

FAGUNDES, G. M. Contribuição ao estudo da vascularização arterial do rim em suínos (Sus scrofa domestica Linnaeus, 1758). 1984. 97 f. Tese (Doutorado em Anatomia dos Animais Domésticos). Universidade de São Paulo, Faculdade de Medicina Veterinária e Zootecnia, São Paulo, 1984.

FERREIRA, N. Contribuição ao estudo dos elementos vasculares, arteriais e venosos do hilo renal em suínos (Sus scrofa scrofa). Rev. Fac. Med. Vet. Zootec. Univ. S. Paulo, v.12, p. 722, 1975.

MACHADO, G. V. et al. Aspectos anatômicos das artérias renais em queixadas (Tayassu pecari Link, 1795). R. Bras. Ci. Vet., v. 7, n. 1, p. 5-8, 2000a.

MACHADO, G. V. et al. Comportamento anatômico das artérias renais em catetos (Tayassu tajacu Linnaeus, 1758). Veterinária Notícias, v. 6, n. 1, p.7-25, 2000 b.

MIGLINO, M. A. et al. Sobre a vascularização renal de ovinos (Ovis aries Linnaeus, 1758) da raça Ideal. In: CONGRESSO BRASILEIRO DE ANATOMIA, 14., 1985, Vitória. Anais... Vitória: SBA, 1985, p. 85.

NEVES W.C., et al.,1993. Estudo dos elementos vasculares, arteriais e venosos, hilares e extra-hilares dos rins da cutia dourada (Dasyprocta aguti). Anais $16^{\circ}$ Congr. Bras. Anatomia, São Paulo, p.154.

PEDUTI-NETO, J. Contribuição ao estudo dos elementos vasculares, arteriais e venosos, do hilo renal, em ovinos da raça Merino. Rev. Fac. Med. Vet. Zoot. Univ. São Paulo, v. 13, n. 1, p. 67-83, 1976.

PRADA, J. C. 2004. Javali: Origem. Disponível em <http:// www.jcprada.com.br/home/historico/origem.htm>. Acesso em 01 dezembro de 2004.

ROMAGNOLLI P., MACHADO G.V., MIGLINO M.A. Arterial segmentes of kidneys in white-lipped peccaries (Tayassu pecari Link, 1795). Braz. J. Vet. Res. Anim. Sci. 40(Supl.)p.205-212, 2003.

SALVINI T.F., FERNANDES W.A. \& OLIVEIRA A. 1981. Contribuição ao estudo dos elementos vasculares arteriais e venosos, do hilo renal de ratos (Rattus norvegicus). Anais $13^{\circ}$ Congr. Bras. Anatomia, Belo Horizonte, p.65.

SAMPAIO, M. A. P. P. et al., Intrarenal arterial distribution in swine. . In: CONGRESSO BRASILEIRO DE ANATOMIA, 17, Fortaleza, 1996. Anais do $\mathbf{1 7}^{\circ}$ Congresso Brasileiro de Anatomia, Fortaleza, Brasil, p.93. 1996. 
SOUZA, A.; BORELLI, V.; FERREIRA, N. Contribuição ao estudo dos elementos vasculares arteriais e venosos do hilo renal, em suínos da raça Landrace. Rev. Fac. Méd. Vet. Zootec. Univ. S. Paulo, v. 21, n.1, p. 27-35, 1984.

DYCE, K. M. et al., Tratado de Anatomia Veterinária, Guanabara Koogan, 2 ed. , p. 140$141,1997$.

SOUZA, A.; BORELLI, V.; FERREIRA, N. Contribuição ao estudo dos elementos vasculares, arteriais e venosos, do hilo renal em suínos da raça Landrace. Revista da Faculdade de Medicina Veterinária e Zootecnia da Universidade São Paulo, v. 21, p. 27-35, 1984.

SOUZA, N. T. M. Segmentos anatomocirúrgicos arteriais do rim em suínos (Sus scrofa domesticus Linnaeus, 1758). 1997. 72 f. Dissertação (Mestrado em Anatomia dos Animais Domésticos) - Universidade de São Paulo, Faculdade de Medicina Veterinária e Zootecnia, São Paulo, 1997.

VILARTA R., et al., 1982b. Contribuição ao estudo dos elementos vasculares, arteriais e venosos, do hilo renal em camundongos (Mus musculus). Anais $1^{a} \mathrm{Sem}$. Veterinária, Faculdade de Medicina Veterinária e Zootecnia, USP, São Paulo, p.17. 